\title{
Perls' Assay To Detect Iron Uptake In The Intestine Of Embryonic Seahorse, Hippocampus barbouri
}

\author{
A.A. Herrera*, and M. R. Bajar* \\ *Institute of Biology, College of Science, University of the Philippines, Diliman Quezon City \\ Iron is a vital micronutrient for teleost fish, being an integral component of the metalloproteins, \\ which are involved in cellular respiration and oxygen transfer.
}

This study aimed to provide baseline information on the iron uptake of an endangered species, the seahorse Hippocampus barbouri. Furthermore, this study determined where specifically in the gut are the particles detected. Information from this study is important for the formulation of a potential feed for the growth and development of $H$. barbouri, which is necessary for the laboratory culture of these endangered species.

Pregnant males were dissected and the exposed brood pouch was emptied of embryonic seahorses. From these embryonic seahorses, gut sections were prepared by standard parraffin technique. The deparaffinized sections were then subjected to Perls' Technique for detection of iron, a method that involves staining with nuclear fast red and potassium ferrocyanide.

In this study, it was determined that iron is present in the gut of the embryonic H. barbouri The iron is present in its ferric state $\left(\mathrm{FE}^{3+}\right)$. It was also noted that the iron deposits were observed only in the late term of the seahorse's embryogenesis. All other embryonic stages exhibited no reaction with Perls' Technique. No definitive proof has been presented in so far as to the presence of a gene coding for an iron transporter in fish is concerned. The acquisition of iron from the diet and across the intestines in teleost fish is widely assumed to occur in a similar mechanism as that with yeast and mammals. The iron deposits were observed near the tips of the villi. Our findings strengthen and further support previous studies suggesting that the export of iron in fish occurs at the enterocytes lining the intestinal villi.

\section{References}

[1] O. Andersen, Fish. Physiol. Biochem. 16 (1997) 233.

[2] N.R. Bury et al., The Journal of Experimental Biology. 204 (2001) 3779.

[3] A.T. Mckie, Mol. Cell. 5 (2000) 299. 


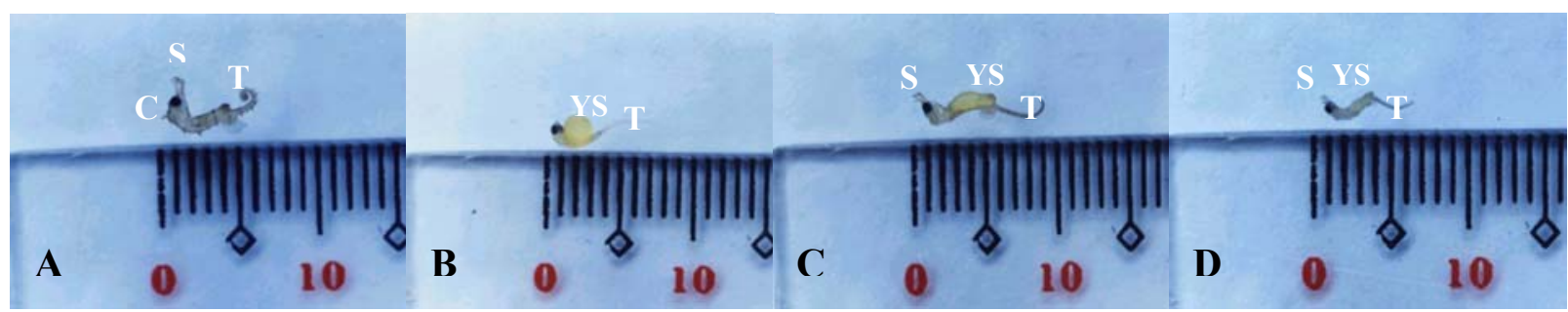

Figure 1. The Barbour's seahorse, Hippocampus barbouri. (A) a six millimeter fry (B) a seven millimeter embryonic seahorse in its early term (C) a nine millimeter embryonic H. barbouri in its middle term (D) a 6.5 millimeter embryonic H. barbouri in its late term, showing regressed yolk sac (Crown-C, Tail-T, Snout-S,YolkSac-YS).

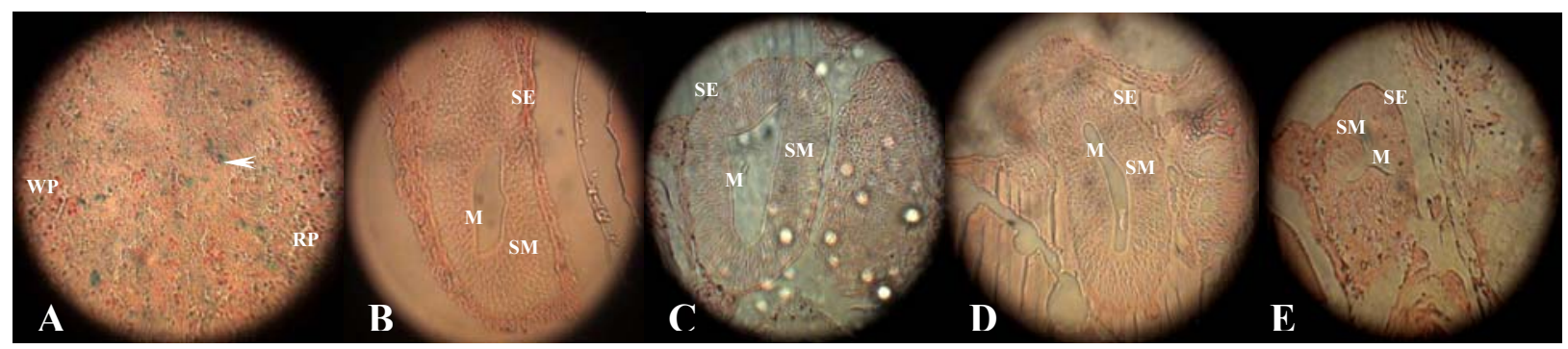

Figure 2. (A) Positive control for Perls' Technique. Embryonic Hippocampus barbouri showing Prussian blue coloration in its spleenic tissues for color comparison. (White Pulp - WP, Red Pulp - RP) (B) Early term of the embryonic middle gut of $H$. barbouri, showing no remarkable coloration. (C) Negative control for early term (D) middle term exhibiting negative reaction to Perls' Staining. (E) Negative control for middle term exhibiting no reaction to Perls' Staining. (Mucosa - M, Submucosa - SM, Developing Muscle - DM Serosa - SE) (200x)

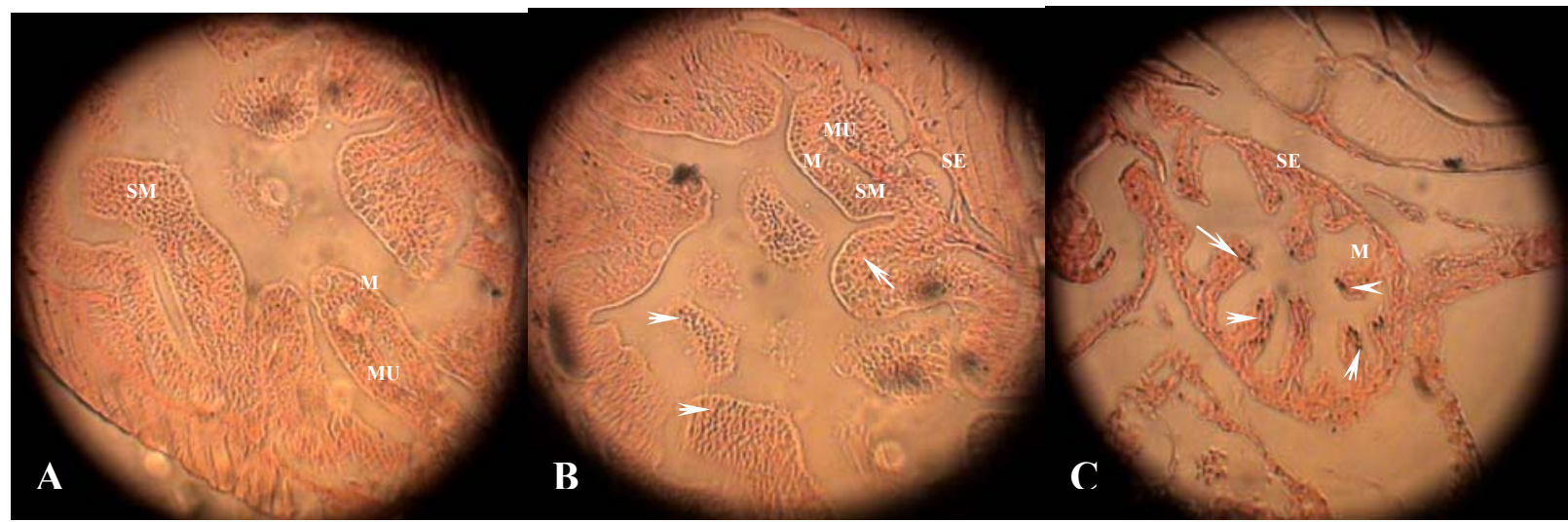

Figure 3. (A) negative control for late term (B) Late term of the embryonic middle gut of H. barbouri, positive for very minimal Prussian blue traces (arrows). (C) Late term of the embryonic middle gut of $H$. barbouri, showing a significant amount of Prussian blue deposits (arrows) in the tissues. (Mucosa - M, Submucosa - SM, Serosa - SE, and Muscularis - MU) (200x) 